*For correspondence: kblackw1@gmu.edu (KTB)

\title{
Synaptic plasticity is predicted by spatiotemporal firing rate patterns . and robust to in vivo-like variability
}

${ }_{4}$ Daniel B. Dorman ${ }^{1}$ and Kim T. Blackwell ${ }^{1,2 *}$

${ }_{5} \quad{ }^{1}$ Interdisciplinary Program in Neuroscience, George Mason University; ${ }^{2}$ Department of

- Bioengineering, Volgenau School of Engineering, George Mason University

8

9

\begin{abstract}
Synaptic plasticity, the experience-induced change in connections between neurons, underlies learning and memory in the brain. Most of our understanding of synaptic plasticity derives from in vitro experiments with precisely repeated stimulus patterns; however, neurons exhibit significant variability in vivo during repeated experiences. Further, the spatial pattern of synaptic inputs to the dendritic tree influences synaptic plasticity, yet is not considered in most synaptic plasticity rules. Here, we address the sensitivity of plasticity to trial-to-trial variability and delineate how spatiotemporal synaptic input patterns produce plasticity with in vivo-like conditions using a data-driven computational model with a calcium-based plasticity rule. Using in vivo spike train recordings as inputs, we show that plasticity is strongly robust to trial-to-trial variability of spike timing, and derive general synaptic plasticity rules describing how spatiotemporal patterns of synaptic inputs control the magnitude and direction of plasticity. Specifically, a high temporal input firing rate to a synapse late in a trial correlated with neighboring synaptic activity produces potentiation, while an earlier, moderate firing rate that is negatively correlated with neighboring synaptic activity produces depression. Together, our results reveal that calcium dynamics can unify diverse plasticity rules and reveal how spatiotemporal firing rate patterns control synaptic plasticity.
\end{abstract}

\section{Introduction}

Synaptic plasticity - the activity-dependent modification of synaptic strength-is widely hypothesized as the neural substrate of learning and memory throughout the brain (Takeuchi et al., 2014). For instance, synaptic plasticity in mammalian striatum (Perrin and Venance, 2019), cortex (Buonomano and Merzenich, 1998), hippocampus (Martin and Morris, 2002), and amygdala (Bocchio et al., 2017) have been linked to procedural, sensorimotor, associative, and emotional learning and memory, respectively. Learning requires that repeated experiences produce a stable, persistent change in synaptic connections which in turn produce stable neural activity and behavioral responses (Abraham and Robins, 2005; Josselyn and Tonegawa, 2020). In vivo experiments have revealed changes in synaptic strength, and generation, elimination, growth or shrinkage of dendritic spines (sites of synaptic input) (Fisher et al., 2017; Trachtenberg et al., 2002; Winnubst et al., 2015; Zhang et al., 2015). However, evidence for stable synaptic changes in response to repeated stimuli primarily comes from in vitro brain-slice experiments with precisely repeated input stimuli patterns, which reveal that stimulus timing, frequency, and synaptic location can control development of long term potentiation (LTP) or long term depression (LTD) (Sjöström et al., 2001; van Rossum et al., 2000; Caporale and Dan, 2008; Lovinger et al., 1993; Hawes et al., 2013). Yet, it is unclear if 
in vitro plasticity discoveries that used precise stimulation patterns are reproducible in the highly variable neural activity conditions in vivo during natural learning and behavior. Indeed, one of the great unsolved questions in neuroscience is whether stable, long-lasting synaptic plasticity occurs in vivo given variable neural activity, and, if so, how robust is synaptic plasticity to variability in response to repeated sensory stimuli and behaviors?

Variability and noise are prominent throughout the brain (Faisal et al., 2008). For instance, cortical neurons exhibit significant trial-to-trial variability in vivo in response to the same repeated external sensory stimulus (Shadlen and Newsome, 1998; Stevens and Zador, 1998). Trial-to-trial variability includes variance in the timing of individual spikes, as well as variance in firing rate over time. Thus, a given postsynaptic neuron could experience variability in the timing and frequency at each of its thousands of synaptic inputs, which would together produce (in addition to cell-intrinsic sources of variability) highly variable output spiking of the postsynaptic neuron. For a postsynaptic neuron to become potentiated or depressed in response to a specific stimulus represented by a subset of its synaptic inputs, plasticity must be robust to variance in the signal (spiking of that particular subset of inputs) as well as variance in the noise (the other synaptic inputs not related to a stimulus). In the in vitro case, not only is presynaptic and postsynaptic spike timing much less variable during repetition, but also the spatial pattern on the dendritic tree of the repeatedly activated synapses is likely less variable in vitro as well.

Experiments suggest that spatial organization of synaptic inputs on the dendritic tree are important for plasticity. For instance, in vitro, cortical synaptic plasticity has been shown to depend not only on rate and timing, but also on cooperativity of inputs (Sjöström et al., 2001), yet it is unclear how in vivo-like variability may affect each of these factors. Additionally, the spatial organization of synaptic inputs on the dendritic tree can affect plasticity by cooperativity among clustered inputs in vitro (Brandalise et al., 2016; Kastellakis et al., 2015; Sjöström et al., 2008; Weber et al., 2016; Golding et al., 2002). Yet, the effect of variability on spatial cooperativity under in vivo-like conditions is unclear, as trial-to-trial variability may affect different spatial patterns, potentially producing cooperative plasticity one trial but not another.

Intracellular calcium elevation is required for most forms of synaptic plasticity, and the spatiotemporal dynamics of the calcium signal-its peak, duration, and location-can determine the occurrence and direction (potentiation or depression) of plasticity (Evans and Blackwell, 2015; Nevian and Sakmann, 2006; Zucker, 1999). In spiny projection neurons (SPNs) of the striatum—input nucleus of the basal ganglia and the focus of this paper-calcium signaling may provide an eligibility trace for corticostriatal plasticity (either LTP or LTD) underlying reinforcement learning for goaldirected or habitual behavior (He et al., 2015; Kreitzer and Malenka, 2008). We have previously shown that synaptic calcium transients are highly sensitive to spatiotemporal patterns of synaptic input and can encode synapse-specificity and cooperativity of neighboring synaptic activity in an experimentally-validated biophysical SPN model with dendritic branches (Dorman et al., 2018). We have also shown that a calcium based plasticity rule with dual amplitude- and duration-thresholds can predict LTP and LTD outcomes of several in vitro plasticity experiments (Uędrzejewska-Szmek et al., 2017). Together, our prior works suggests that our calcium based plasticity rule, implemented in biophysical models with realistic morphology, could predict whether plasticity is robust to spatiotemporal trial-to-trial variability and which specific spatiotemporal patterns produce LTP or LTD.

Here, we investigate computationally the effect of spatiotemporal synaptic activity patterns and trial-to-trial variability to predict if persistent synaptic plasticity occurs with in vivo-like synaptic variability. We find that persistent plasticity is robust to trial-to-trial variability, and we also demonstrate that the spatial pattern of activity on the dendritic branch is critical for determining whether LTP or LTD occurs. 


\section{Results}

\section{Data-driven SPN model exhibits calcium-based synaptic plasticity for in vivo-like inputs}

To investigate the effects of in vivo-like corticostriatal synaptic inputs on corticostriatal plasticity, we created a realistic biophysical SPN model (Prager et al., 2020) with a calcium-based plasticity rule (Jędrzejewska-Szmek et al., 2017) and simulated in vivo-like synaptic input patterns. The multicompartment, multi-ion channel model was optimized to fit electrophysiological data using an extended version of a parameter optimization algorithm we developed (Jedrzejewski-Szmek et al., 2018; Dorman and Blackwell, 2021). The model reproduced the characteristic electrophysiological responses of SPNs.

Trial-to-trial variability is regularly observed in in vivo spike train recordings but the effect of variability on plasticity is unclear. To simulate synaptic plasticity in response to trial-to-trial variability, we obtained spike train recordings from the anterior lateral motor cortex from a published dataset ( Li et al., 2015) from which we constructed synaptic inputs to the model. An initial single trial of corticostriatal inputs was constructed from all the spike trains of 22 behaviorally similar experimental trials to generate sufficient synaptic drive while maintaining, as much as possible, potential withintrial correlations between neurons present in the dataset. The initial one-second trial (shown as raster plot and peri-stimulus time histogram in Figure $1 \mathrm{~A}, \mathrm{~B}$ ) produced depolarization and spiking in the SPN (Figure 1C) with a firing rate consistent with in vivo observations.

The first question addressed is whether a calcium-based synaptic plasticity rule that was derived to explain STDP data is sufficiently general to produce synaptic plasticity in response to spatiotemporally distributed synaptic inputs. To determine whether this calcium-based plasticity rule would predict plasticity for in vivo like conditions, we simulated synaptic weight changes in response to trial-to-trial variability. We used our calcium-based plasticity rule that can reproduce results from several spike-timing dependent plasticity experiments on SPNs in vitro (Jędrzejewska-Szmek et al., 2017). Crucially, this rule is entirely based on spine calcium dynamics, not relative spike timings, so it is a general rule that encompasses both frequency-based and spike-timing plasticity rules.

Cortical spike trains indeed produced synaptic plasticity with our calcium-based plasticity rule. We examined the spine calcium concentration and the synaptic weight of every synapse in response to a single 1-second trial of randomly distributed in vivo spike trains. We found that, at the end of a single trial, some synapses exhibited potentiation, some exhibited depression, and others exhibited no change (Figure 2-example synapses; Figure 3-all synapses), with most exhibiting little change. These results show that a calcium-based plasticity rule determined from in vitro data can produce plasticity with spatially distributed in vivo-like synaptic input conditions.

\section{Synaptic plasticity is highly robust to trial-to-trial variability}

During repeated behaviors, cortical neurons exhibit significant levels of trial-to-trial variability, yet it remains unclear how this variability affects synaptic plasticity. For synaptic plasticity to serve as the basis of learning, it should be robust to naturally observed variability in neuron spiking. However, many plasticity experiments use highly regular, precisely repeated stimulus patterns. To bridge the gap between in vitro plasticity findings due to precisely repeated stimuli and in vivo plasticity with spatiotemporally dispersed inputs and trial-to-trial variability, we simulated the response to 10 repeated trials, with varying levels of trial-trial variability. This is analogous to 10 behavioral learning trials during which striatal neurons receive variable cortical input from trial to trial.

For every level of trial-to-trial variability we simulated, a subset of synapses exhibited robust weight change at the end of 10 repeated trials. As shown in Figure 4A, synaptic weight over time consistently accumulates potentiation or depression for a subset of synapses regardless of variability level. This robust weight change also is observed when variability is introduced by randomly moving spikes to different trains (Figure 4- supplementary figure 1). These results predict that synaptic weight change is robust to high levels of trial-to-trial spike time variability, suggesting that 


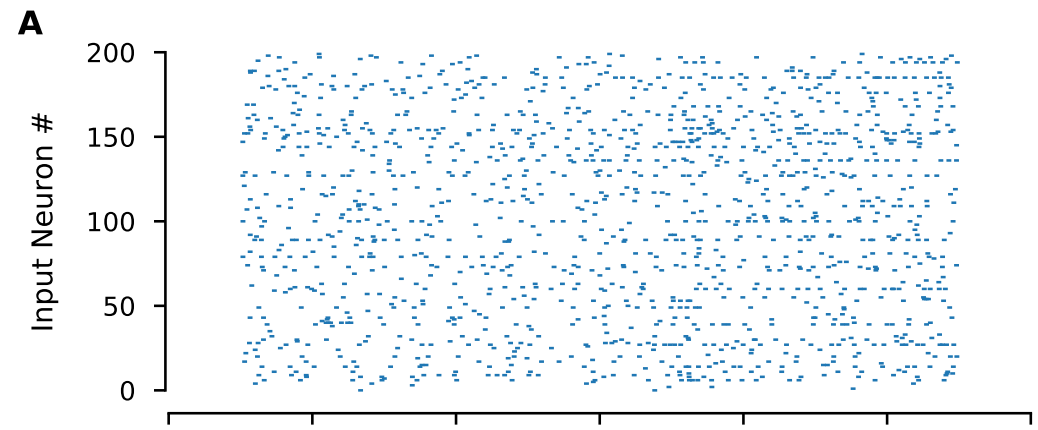

$\mathbf{B}$

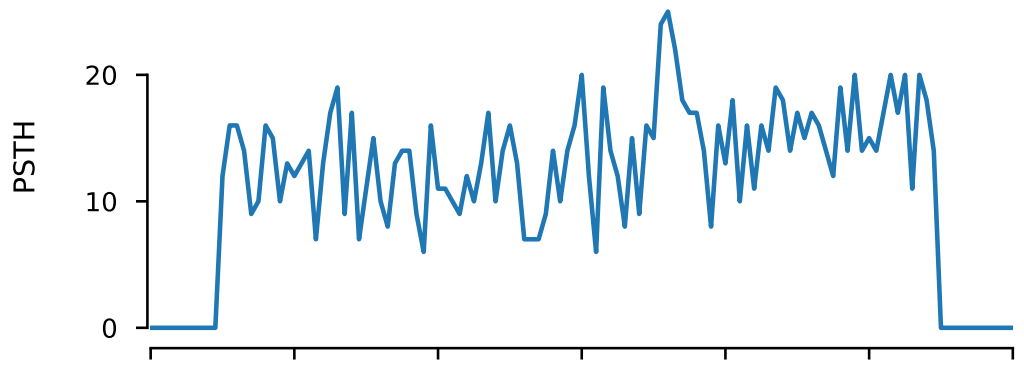

C

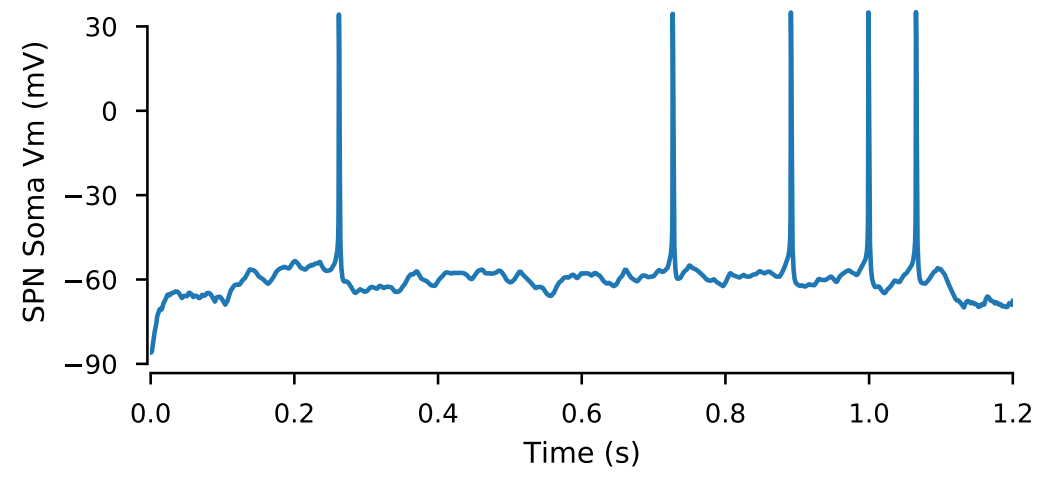

Figure 1. In vivo-like inputs constructed from cortical spike trains produce spiking in SPNs

A. Raster plot shows spike times for each cortical input in the model, constructed from in vivo spike train recordings. B. Peri-stimulus time histogram of the above raster plot (spike counts per $10 \mathrm{~ms}$ bin) C. Somatic membrane potential of the SPN model showing spiking output induced by cortical input.

in vivo variability does support synaptic plasticity.

Though synaptic weight change persisted across levels of trial-to-trial variability, the magnitude of weight change at the end of an experiment was reduced for increasing levels of variability. Figure $4 \mathrm{~B}$ shows ending synaptic weight as a function of trial-to-trial variability, demonstrating that high variability reduces the magnitude of depression, but not potentiation, both for jittered and moved spikes (Figure 4-supplementary figure 2). These results suggest that variability in spike timing primarily effects the magnitude of plasticity but rarely the direction (potentiation or depression).

\section{Plasticity of a single synapse is only partially predicted by its presynaptic activity} Individual synapses receive wide ranges of presynaptic input patterns and exhibit a broad range of synaptic plasticity outcomes. Which properties of synaptic input patterns, that are potentially modulated by trial-to-trial variability, predict the magnitude and direction of synaptic plasticity? To investigate this question, we first asked whether the total presynaptic spike count for a given 
A

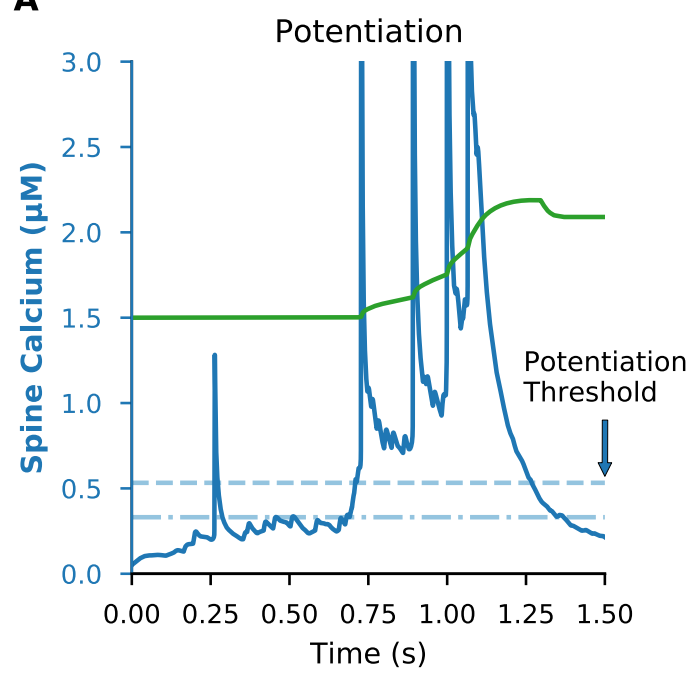

B

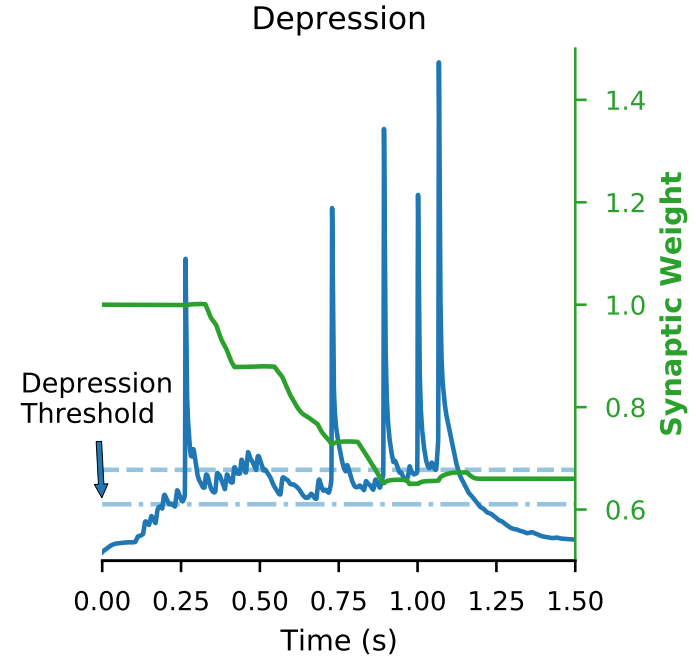

Figure 2. Calcium-based plasticity rule produces both potentiation and depression

A calcium-based plasticity rule was implemented with dual amplitude and duration thresholds. LTD required that spine calcium concentration exceed the amplitude threshold (dot-dashed line) of $0.33 \mu \mathrm{M}$ for greater than $28 \mathrm{~ms}$, while LTP required that spine calcium concentration exceed a higher amplitude threshold (dashed line) of $0.53 \mu \mathrm{M}$ for at least $3.3 \mathrm{~ms}$. Example traces are shown of a synapse that potentiates (A) or depresses (B) following a single trial. Blue lines show spine calcium concentration (with left y-axis) and green lines show synaptic weight (with right $y$-axis) for each example synapse.

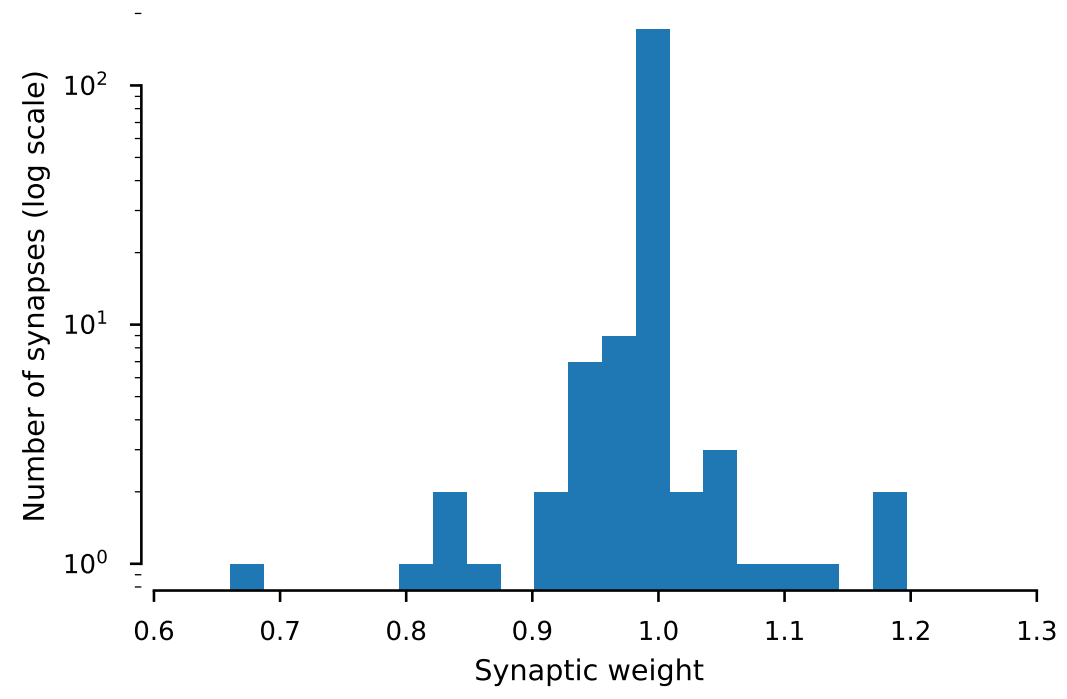

Figure 3. Distribution of synaptic weights after initial trial

Histogram shows the distribution of synaptic weights for all synapses following a single trial (note log scale of $y$ axis). All weights were initialized at 1 , and post-trial weights greater than 1 are potentiation while weights less than 1 are depression.

synapse predicted that synapse's weight at the end of 10 trials (ending weight). We compared ending weight of every synapse to its total presynaptic spike count across all 10 trials for experiments at different levels of trial-to-trial variability (Figure 5). We found a general pattern that synapses with low presynaptic spike counts exhibited little to no synaptic weight change; synapses with a moderate presynaptic spike count tended to exhibit depression; and synapses with a high presynaptic spike count exhibited potentiation. However, a wide range of ending synaptic weights was observed for synapses with moderate presynaptic spike counts, and further, these synapses were 
bioRxiv preprint doi: https://doi.org/10.1101/2021.08.03.454974; this version posted August 5, 2021. The copyright holder for this preprint (which was not certified by peer review) is the author/funder, who has granted bioRxiv a license to display the preprint in perpetuity. It is made available under aCC-BY-NC 4.0 International license.

A

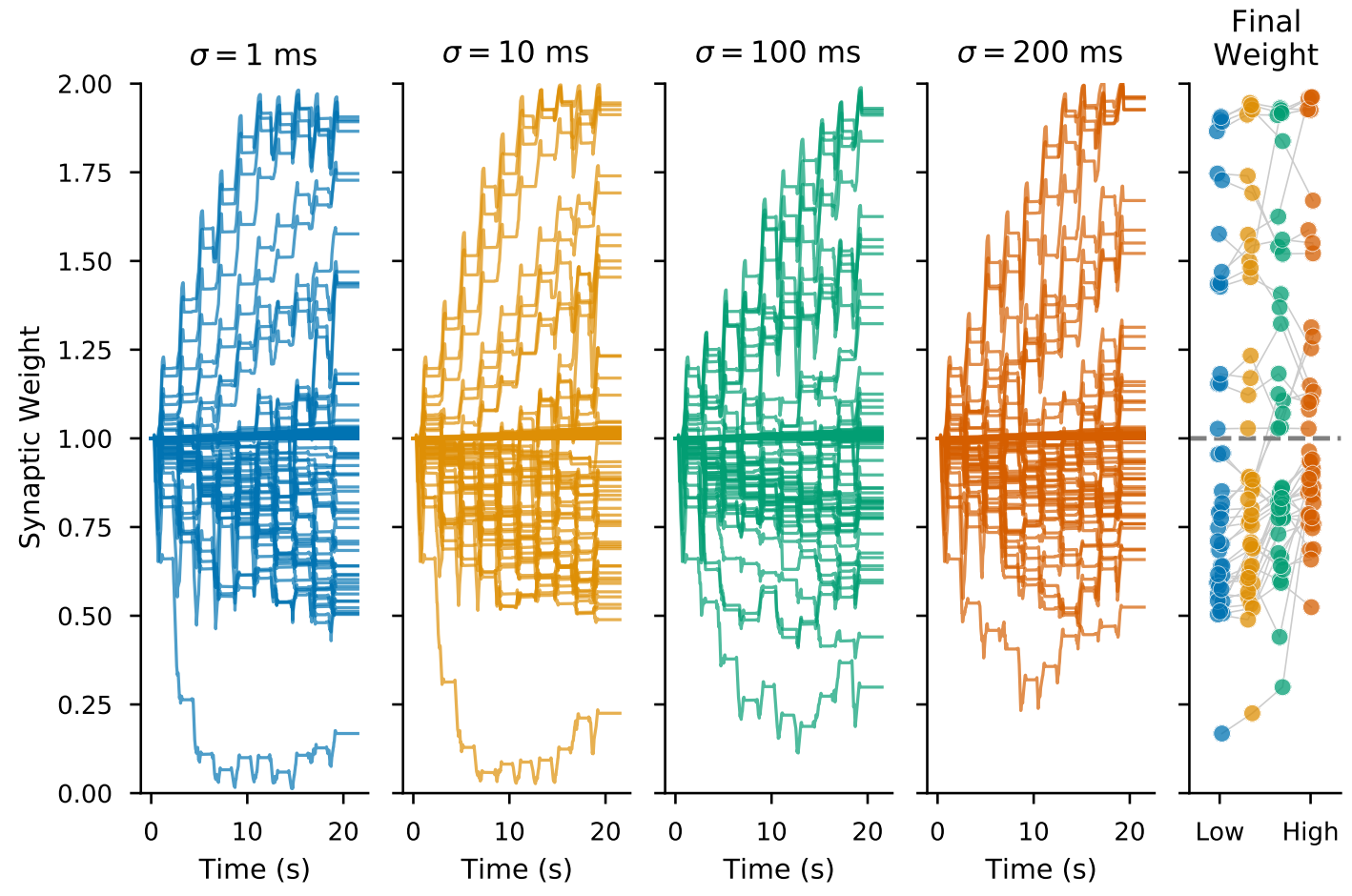

B
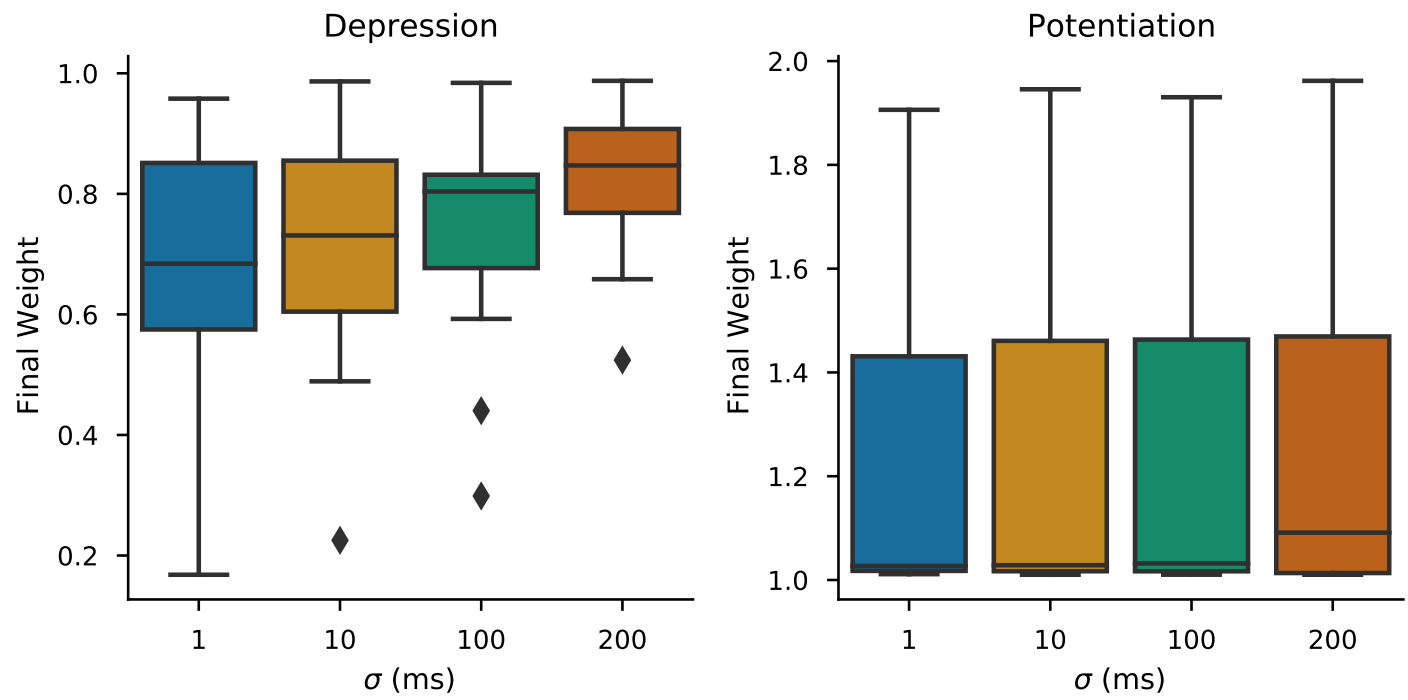

Figure 4. Synaptic plasticity is robust to trial-to-trial variability

(A) The weight of every synapse over a full 10-trial experiment is shown for different trial-to-trial variability conditions, with the sigma value corresponding to the standard deviation of random jitter of spike times. (Right) Ending weight of each synapse is shown for each variability condition (synapses with near zero weight change excluded for visualization). (B) Distribution of final weights grouped by potentiation and depression shows that variability reduces synaptic depression magnitudes, but has little effects on the distribution of potentiation magnitudes. Correlation of ending synaptic weight versus variability was significant for depressing synapses $(R=0.306, p=0.0006, N=121$ events), but not for potentiating synapses $(R=0.054, p=0.510$, $\mathrm{N}=148$ events) 


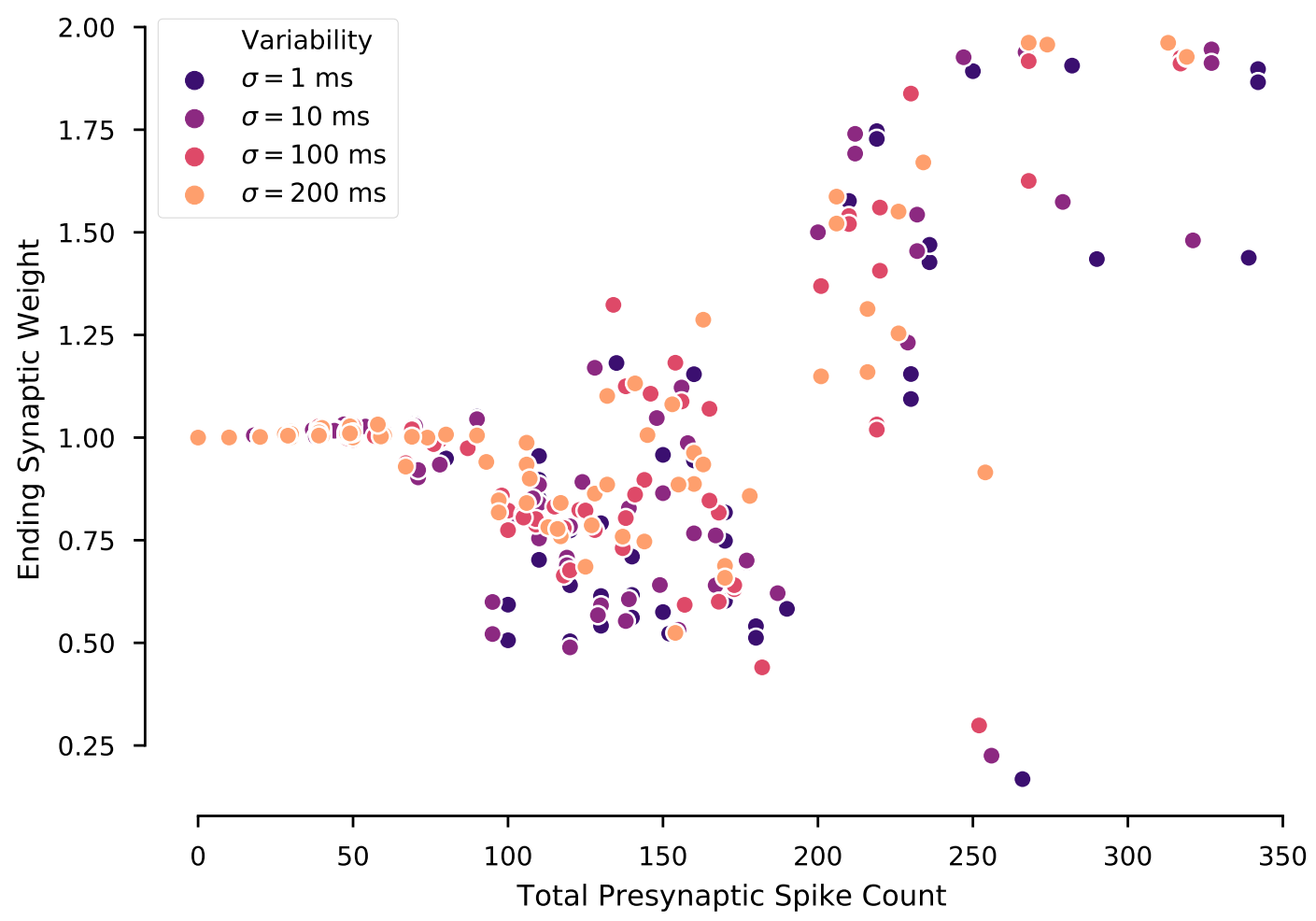

Figure 5. Ending synaptic weight is partially predicted by total presynaptic spike count per synapse Ending synaptic weight of each synapse is plotted versus its total presynaptic spike count across all 10 repeated trials, with experiments separated by the level of trial-to-trial variability. Ending weight exhibits no change for low spike counts, tends toward depression for intermediate spike counts, and exhibits potentiation for high spike counts. This trend is consistent regardless of trial-to-trial variability; however, for intermediate spike counts the ending weight is highly variable.

most affected by trial-to-trial variability. The synaptic weight was not correlated with the synapse's distance to the soma, except for the lower levels of variability (Figure 5-Supplementary Figure 1). These results suggest that while spike count alone is a significant factor in predicting synaptic weight change, other spatiotemporal factors may also be important, and these factors may be significantly affected by trial-to-trial variability.

\section{Plasticity of a single synapse is affected by presynaptic temporal firing rate pattern}

As total presynaptic spike count alone did not completely predict ending synaptic weight per synapse, we next investigated the effect of the temporal pattern of presynaptic firing rate on synaptic plasticity. Though spike count per synapse was consistent for experiments, the trial-to-trial variability introduced changes in spike timing that altered the time-varying instantaneous presynaptic firing rate for each synapse. To identify whether instantaneous firing rate over the course of a single trial was associated with potentiation, depression, or no-change, we computed a weight-change triggered average presynaptic firing rate. This weight-change triggered average was computed by binning trials and synapses based on the magnitude of synaptic weight change following an individual trial, computing the instantaneous presynaptic firing rate vs. time for each synapse and trial, and averaging across synapse-trials within each bin.

Our results show that synapses that strongly potentiate exhibit a weight-change-triggered average presynaptic firing rate with a high peak firing rate late in the trial. In contrast, synapses that strongly depress exhibit an earlier peak firing rate or a moderate sustained presynaptic firing rate. Synapses with little or no weight change exhibit a low presynaptic firing rate (Figure 6A). This pattern was also observed when variability was introduced by moving spikes between trains (Fig- 


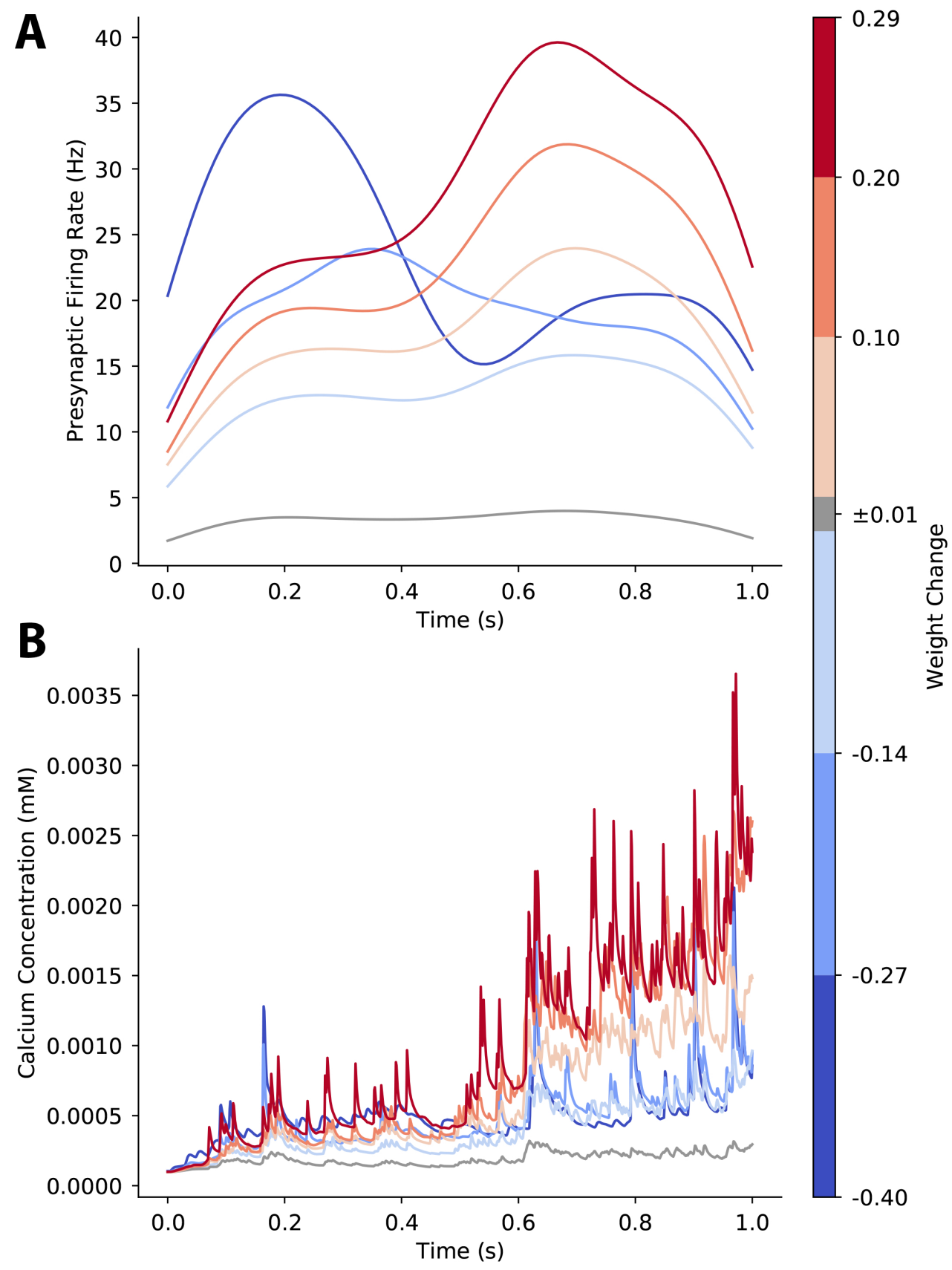

Figure 6. Temporal pattern determines direction of plasticity as shown by weight-change-triggered-average

A. For each synapse on each trial, we computed the instantaneous firing rate of its presynaptic input activity and grouped synapse-trials into bins based on the size of the synaptic weight change that occurred following a single trial. Then, we averaged across the instantaneous firing rate of each bin. Late and high peak firing rates lead to LTP, while earlier peak firing rate or moderate firing rate leads to LTD. B. For each synapse on each trial, we computed the calcium concentration for each synapse-trial and averaged across the calcium concentration for each weight change bin. Calcium concentration is higher during the second part of the trial, both for potentiating and depressing synapses.

ure 6-supplementary figure 1A). We also calculated the weight-change-triggered average calcium concentration, to assess how presynaptic firing dynamics were translated into calcium elevations. As shown in Figure 6B (and Figure 6-supplementary figure 1B), synapses that potentiate have higher calcium concentration than synapses that depress, and synapses that strongly potentiate 


\begin{abstract}
have the highest calcium. Though the largest differences are in the second half of the trial, weight change dependent differences in calcium concentration for synapses that depress are more apparent in the first half of the trial. These results suggest that input timing within a trial, which affects peak instantaneous firing rate and calcium concentration, is a critical factor for the direction and amplitude of synaptic weight change. Though temporal pattern discriminates strong LTP from strong LTD, the temporal patterns for moderate plasticity are not as clear. Thus, next we evaluated the role of spatial patterns of synaptic input.
\end{abstract}

\title{
Plasticity of a single synapse is affected by neighboring synaptic activity
}

Prior work has shown that synaptic plasticity can be affected by spatiotemporally cooperative synaptic activity - that is, multiple synapses on the same dendritic branch active within a limited time window (Govindarajan et al., 2011; Legenstein and Maass, 2011; Cichon and Gan, 2015; Brandalise et al., 2016; Magó et al., 2020; Weber et al., 2016; Losonczy et al., 2008). Our prior work has shown that spatiotemporal activity patterns have nonlinear, spatially specific effects on calcium transients in dendrites and spines (Dorman et al., 2018). Thus, it is likely that nearby synaptic activity can cooperatively influence plasticity in our calcium-based model. As shown in figure 5, a given synapse's presynaptic firing rate, alone, is not sufficient to fully determine its synaptic weight change. Therefore, we next investigated the cooperative effect of neighboring synaptic activity on weight change at each synapse.

We found that synapses which strongly potentiate experience higher neighboring synaptic activity in the dendritic branch than synapses which strongly depress (Figure 7A) for both types of spike train variability (Figure 7-supplementary figure 1). This suggests that cooperative synaptic activity among neighboring synapses within a dendritic branch influences the outcome of plasticity. We averaged across neighboring synapses because no spatial pattern was apparent when considering input to neighboring synapses individually (Figure 7-supplementary figure 2). To further investigate a relationship between direct and neighboring inputs for plasticity, we computed correlation coefficients between each synapse's direct instantaneous firing rate and the combined rate of its neighbors, again binned by plasticity outcome. As shown in Figure 7B, strongly potentiating synapses were more likely to be positively correlated with neighboring activity, while strongly depressing synapses were more likely to be negatively correlated. Together, our results show that both temporal and spatial patterns are critical to synaptic plasticity.

\section{Dynamics of pre-synaptic firing rate predicts synaptic plasticity}

To further assess robustness of the results, we repeated simulations using 1000 different variations of the mapping from spike trains to synapses. Since the change in plasticity is consistent with repeated trials (Fig 4), we simulated a single trial. The weight-change-triggered triggered pre-synaptic firing rate (Figure 7-supplementary figure $3 \mathrm{~A}$ ) reveals that, similar to Fig 6, synapses that strongly potentiate have a transiently high firing rate, though the time of peak firing varies. Regardless of the dynamics of the pre-synaptic firing rate, the calcium concentration (Figure 7- supplementary figure 3B) exhibits a higher concentration later in the trial, as observed in Fig 6B. The firing rate of neighboring synapses (Figure 7- supplementary figure 3C) reveals a much higher firing frequency for neighbors of potentiating synapses than neighbors of depressing synapses. For synapses that exhibited a weight change, the correlation between neighboring synapse firing rate and weight change was 0.1 , averaged across 5 sets of simulations. Though the correlation is low, it is highly significant $(\mathrm{P}<0.0001)$ demonstrating that spatiotemporal firing patterns are key to determining synaptic plasticity.

To quantitatively assess how spatiotemporal pattern of synaptic input controls synaptic plasticity, we used random forest regression to predict synaptic weight change from instantaneous firing rate to the synapse, together with either firing rate of neighboring synapses, correlation between direct input and input to neighboring synapses, or distance of synapse to the soma. The instantaneous firing rate was discretized into a small number of time samples to coarsely represent the 

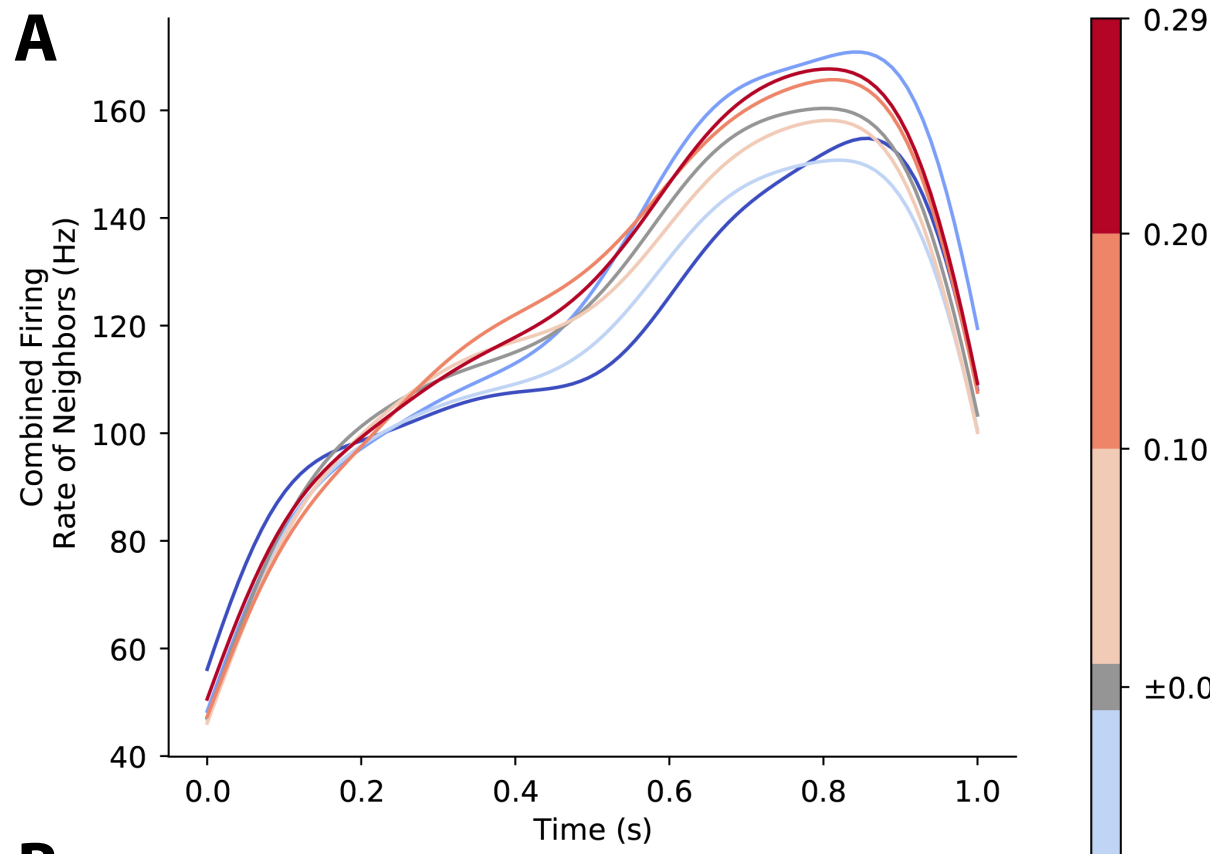

B

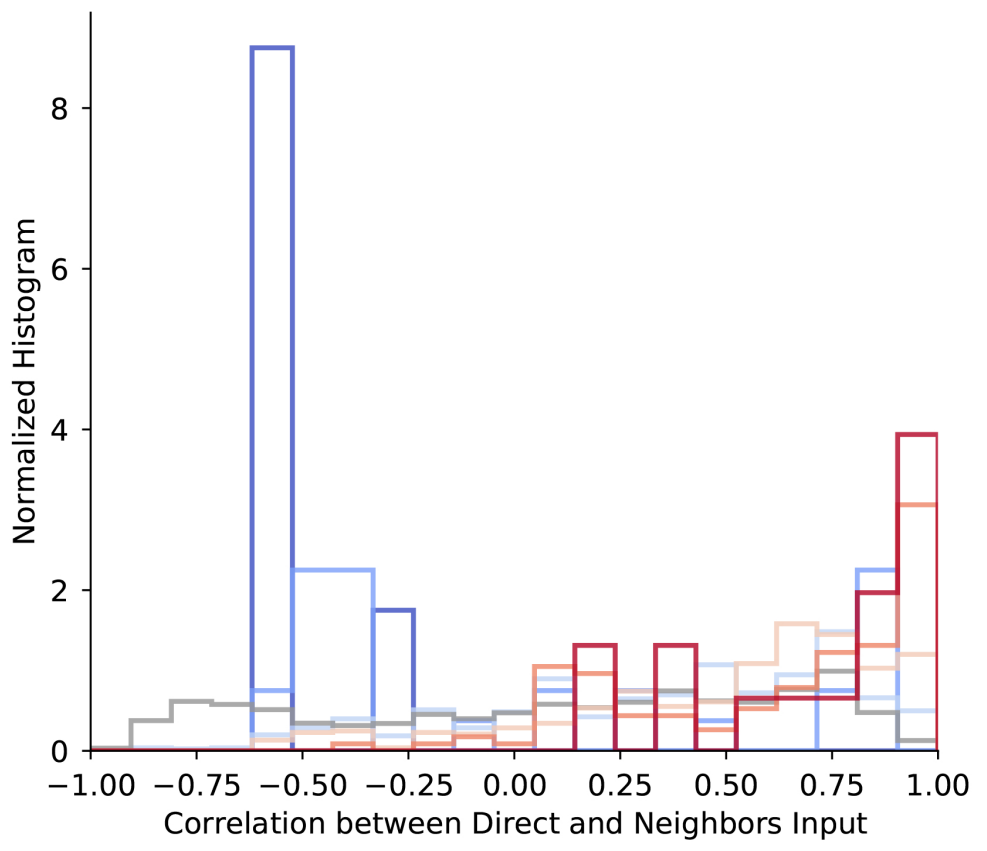

0.10

$\pm 0.01$

Figure 7. Activity of neighboring synapses influences direction of plasticity

A. Neighboring synaptic activity is associated with small difference in strong LTP and LTD. Traces show the combined neighboring synaptic instantaneous firing rate averaged for bins of synaptic weight change following a trial. For every synapse, its 20 nearest neighbors' spike trains were combined and an instantaneous firing rate was computed from the combined spike train. Synapses were then binned by amount of weight change following a trial, and the average neighboring instantaneous rate was computed for each bin. B. Correlation between direct and neighboring synapses influences direction of plasticity. Histograms show the correlation between direct and neighboring presynaptic firing rates binned by plasticity outcome. High positive correlation between direct and neighboring firing rate is associated with LTP, while low correlations are associated with LTD. 
not improve the prediction. The same pattern is observed when variability was introduced by moving spikes between trains (Figure 8- supplementary figure 1A) or using alternative mappings of spike trains to synapses (Figure 8- supplementary figure 1B). In summary, the dynamics of direct input to the synapse is crucial for determining synaptic plasticity, but firing rate of neighboring synapses was not needed, despite the correlation between synaptic weight change and neighboring firing rate, likely due to ability of random forest regression to find temporal features.

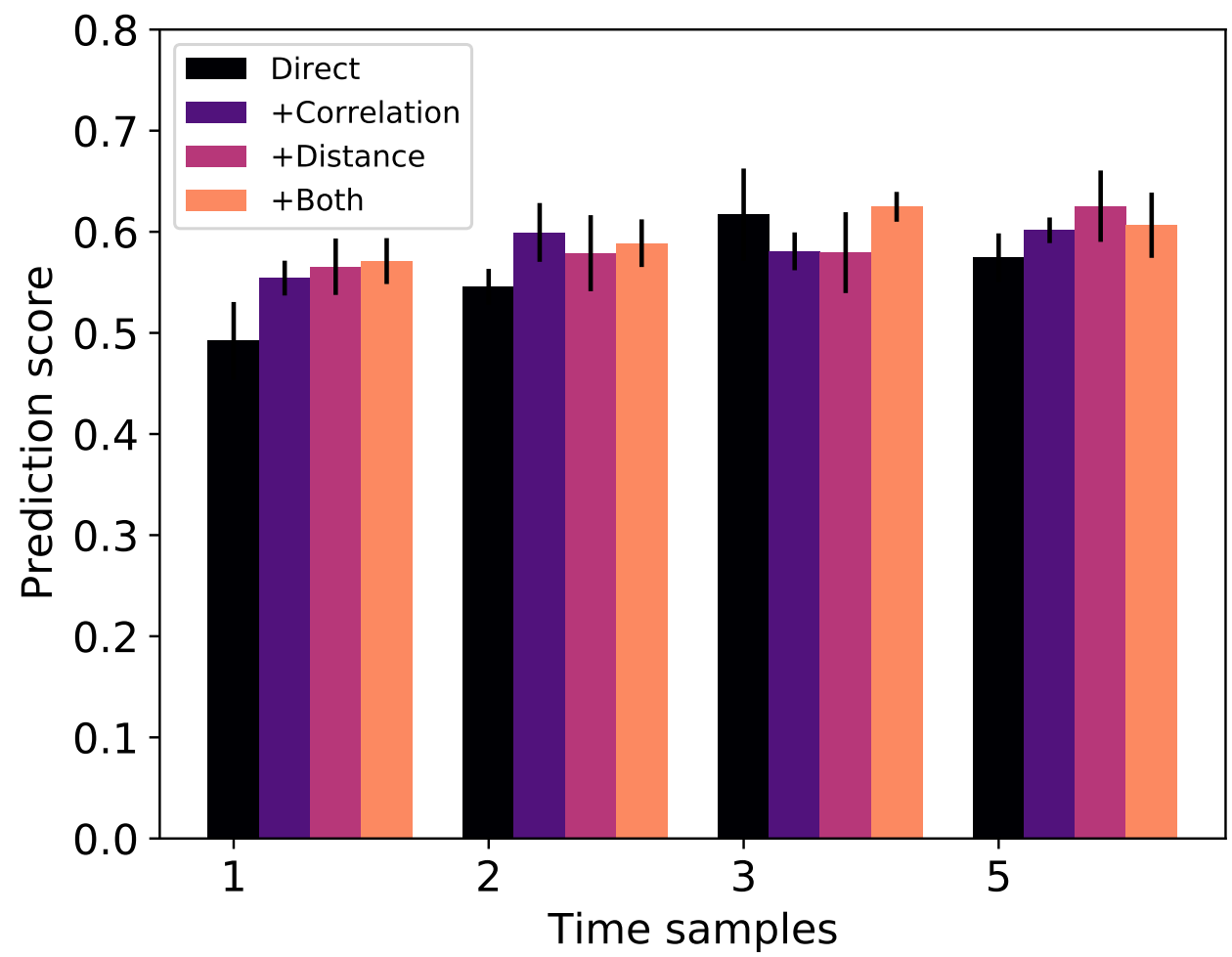

Figure 8. Temporal pattern of input predicts weight change better than mean firing rate Prediction score is the coefficient of determination, $\mathrm{R}^{2}$, of the predicted weight change for the test set. $\mathrm{N}=4$ regressions for each combination of features. Error bars show 1 standard error. ANOVA shows that increasing number of time samples improves the prediction score $(F(3,76)=4.776, P=0.0042)$.

\section{Discussion}

In this study, we addressed how temporal and spatial patterns of in vivo-like synaptic inputs with trial-to-trial variability impact the direction and magnitude of synaptic plasticity. We employed a calcium based plasticity rule in a biologically constrained computational model previously validated on in vitro plasticity protocols to predict plasticity for in vivo-like conditions. We found that synaptic plasticity with in vivo-like activity is robust to trial-to-trial variability. Further, we found that the temporal pattern of synaptic inputs within a trial and the spatial pattern of neighboring synaptic inputs on the dendritic tree controls synaptic plasticity outcomes, with a transient high firing frequency producing strong LTP and a moderate increase in firing frequency producing LTD. The firing frequency of neighboring synapses relates to the plasticity outcome, with correlation or high firing frequency of neighbors producing LTP and lack of correlation or low firing frequency of neighbors producing LTD. Our work provides key insights into the nature of synaptic plasticity in conditions more likely to occur during natural behavior. Together, these results predict that plasticity is highly robust to variable spike timing in the brain and suggest that both temporal and spatial aspects of 
synaptic integration are critical to plasticity.

Our finding that plasticity at a single synapse is influenced by neighboring synaptic activity is consistent with many studies (both in vivo and in vitro) showing relationships between spatial synaptic input patterns and plasticity. For instance, in SPNs in vitro, spatially clustered synaptic inputs can produce supralinear depolarization (termed NMDA-spikes or plateau potentials) and synaptic calcium transients (Plotkin et al., 2011; Dorman et al., 2018; Prager et al., 2020; Du et al., 2017), and synaptic activation of 2-4 neighboring spines at depolarized potentials can produce nonlinear enhancement of spine calcium transients (Carter et al., 2007). Building on these findings, our work predicts that neighboring synaptic interactions can influence the direction and magnitude of corticostriatal synaptic plasticity in vivo, with high neighboring activity producing LTP and low neighboring activity producing LTD. Spatially clustered inputs have been shown to induce synaptic potentiation (termed cooperative LTP) in cortical and hippocampal pyramidal neurons in vitro (Brandalise et al., 2016; Golding et al., 2002; Gordon et al., 2006; Larkum et al., 2009; Losonczy et al., 2008; Makara and Magee, 2013; Schiller et al., 2000; Weber et al., 2016; Magó et al., 2020). Similarities in synaptic integration properties of SPNs and pyramidal neurons (Oikonomou et al., 2014) suggest that our calcium-based plasticity rule could be implemented in models of pyramidal neurons and could account for cooperative LTP observations in vitro and predict in vivo plasticity in pyramidal neurons.

Observations suggest that functional synaptic clustering is a key component in plasticity and learning. For instance, correlated activity in spatially clustered spines has been observed in vivo in pyramidal neurons (Takahashi et al., 2012; Winnubst et al., 2015; Wilson et al., 2016; Kerlin et al., 2018). Further, in vivo calcium transients in neighboring spines of the same dendritic branch correlate with structural potentiation of spines and behavioral learning in motor cortical neurons (Cichon and Gan, 2015). This is consistent with our observation that strongly potentiating synapses had higher synaptic inputs than depressing synapses. However, it is unclear whether functional clustering arises from synaptic connectivity in development, or if activity dependent plasticity can generate functional clusters starting from random connectivity. Our results, which used randomly distributed inputs, suggest that spatial synaptic correlations may emerge with random spatial distribution of inputs, thereby potentiating neighboring synapses that each receive higher than average input and producing functional synaptic clusters. These implications of our results are consistent with another modeling study investigating the impact of synaptic clustering on somatic membrane potential with in vivo-like conditions (Ujfalussy and Makara, 2020), which suggested that global plasticity rules would not be sufficient for formation of synaptic clusters. Ujfalussy and Makara (2020) predicted that local plasticity rules would be necessary, though their model did not implement local plasticity rules to test that prediction. Our calcium-based plasticity rule, which accounts for both local and global plasticity effects, is a method for formation of synaptic clusters, consistent with Ujfalussy and Makara (2020) as well as experimental evidence. The resulting synaptic plasticity accounts for the impact of local dendritic activity, and the potentiation of correlated synapses can produce spatial clustering. Our results also suggest a role for spatial patterns in synaptic depression. Specifically, whereas high correlations among neighboring synapses were associated with potentiation, synapses that depressed had negatively correlated or lower than average neighboring synaptic activity.

This suggests a mechanism for spatially balanced potentiation and depression, which could provide homeostatic balance and prevent runaway potentiation. These implications are consistent with in vivo experiments that have shown spine shrinkage in inactive spines accompanying structural potentiation of nearby spines, suggesting heterosynaptic depression as an important compensatory plasticity mechanism (Oh et al., 2015; El-Boustani et al., 2018). Our results suggest that our calcium-based plasticity rule effectively captures the impact of neighboring synaptic activity on depression to support compensatory plasticity. Predictions from our results could be experimentally tested in vitro using glutamate uncaging to apply temporally correlated or uncorrelated patterns to neighboring dendritic spines, together with whole cell recording to measure LTP 
or LTD and calcium imaging of dendritic spines to relate stimulation patterns with calcium activity and synaptic plasticity.

The distinct temporal patterns that were associated with potentiation or depression have implications for striatal and wider basal ganglia circuit function. Though corticostriatal LTP requires dopamine in addition to calcium elevation (Fisher et al., 2017), prior experimental work has shown calcium-dependent synaptic eligibility traces in SPNs (Shindou et al., 2019). These eligibility traces for corticostriatal LTP exhibit a temporal dependence such that an LTP protocol followed within a few seconds by dopaminergic stimulation produces LTP (Yagishita et al., 2014). This pattern of cortical inputs followed by dopamine is consistent with reinforcement learning, as the rewarding outcome (represented in the striatal dopamine signal) temporally follows the action that produced it (represented in corticostriatal activity). Thus, though our plasticity model doesn't account for dopamine directly, we suggest that our calcium-based plasticity rule accounts for eligibility traces for LTD or LTP and captures the spatiotemporal pattern of corticostriatal activity that, when followed by dopaminergic stimulation, produces plasticity. Further, as dopaminergic activity is consistent with volume transmission (Borroto-Escuela et al., 2018; Zoli et al., 1998), we suggest that our calcium-based plasticity rule provides the spatiotemporal specificity indicating which synapses are eligible for reinforcement when followed by a spatially diffuse dopamine signal.

This study has important implications for plasticity in variable in vivo conditions by showing that precise spike timing is not required. Our work suggests that while plasticity is sensitive to firing rate, it is highly robust to variance in precise spike timing during a trial. In vitro corticostriatal spike-timing dependent plasticity experiments demonstrated that NMDAR-dependent (and spike-timing dependent) LTP is highly sensitive to jittered spike timings, though increasing pairings of presynaptic and postsynaptic stimuli could recover LTP (Cui et al., 2018). Thus, precise spike timing rules could make in vivo plasticity unlikely, given variability of spike timing (Williams et al., 2019). Importantly, our calcium-based plasticity rule is independent of spike timing, though we previously showed it could reproduce in vitro spike timing dependent plasticity results. Thus, we suggest our rule is generalizable to in vivo conditions, and we predict that plasticity in vivo is robust to variable spike timing. Consistent with this implication, other work has shown that cortical and striatal neurons exhibit decreasing trial-to-trial variability in vivo during learning that corresponds to reduced behavioral variability of the learned action, and this reduction is dependent on striatal plasticity (Santos et al., 2015). Our result that potentiation is robust to high trial-to-trial variability suggests that corticostriatal plasticity may occur even with highly variable conditions early in learning and then, by potentiating the relevant synapses, produce decreased variability in striatal spiking with learning.

Our work is consistent with a prior model that predicted that plasticity was not sensitive to spike timing with in vivo like firing patterns (Graupner et al., 2016). Nonetheless, our research is a major advance over that prior work which implemented a plasticity rule based on simplified calcium dynamics in a non-spatial model (Graupner and Brunel, 2012) or derived firing rate plasticity models from simplified calcium dynamics (Lappalainen et al., 2019). Our calcium-based rule is implemented with detailed biophysical models of calcium dynamics in a neuron model that includes dendritic morphology. More importantly, we extend prior work to show that temporal patterns are still important, and that spatial interactions among synapses influence plasticity. We also predict that potentiation and depression are differentially sensitive to both spatiotemporal patterns and trial-to-trial variability.

Our results show that the spatial aspects of synaptic integration may contribute to synaptic plasticity. However, spiking network models that incorporate spike-timing dependent plasticity rules to investigate the effect of plasticity on network activity neglect spatial patterns of synaptic inputs to a single neuron, reducing neurons to point processes (Legenstein et al., 2005; Berthet et al., 2016; Dunovan et al., 2019). We suggest that our calcium based plasticity rule could be used in future work to develop simplified plasticity models incorporating both temporal and spatial effects of synaptic activity. 
A statistical model using temporal and spatial kernels to predict the effect of both direct and neighboring synaptic activity on the synaptic weight of each synapse could be derived from biophysical single neuron models with our calcium based rule and then simulated efficiently in large networks with simplified neuron models. Incorporating this synaptic plasticity rule in large scale simulations of network models of the basal ganglia could then better predict how corticostriatal plasticity supports goal-directed and habit learning and identify potential therapeutic targets for modulating aberrant plasticity in addiction.

\section{Methods}

We developed a biologically-constrained computational SPN model with multiple ion channels identified in SPNs, real dendritic morphology, explicit dendritic spines, sophisticated calcium dynamics, and a calcium-based plasticity rule. Model parameters were determined using our parameter optimization software to fit the model to electrophysiological current injection data (Dorman and Blackwell, 2021). To generate in vivo-like synaptic inputs, we obtained and analyzed anterior lateral motor cortical spike trains from the CRCNS.org repository. The model was simulated with these in vivo spike trains to investigate whether plasticity occurs with in vivo-like activity.

\section{SPN model morphology and passive membrane properties}

A biophysical SPN model we previously published (Dorman et al., 2018) was adapted and translated from the GENESIS simulator format to our declarative format (Blackwell et al., 2021) for the MOOSE simulator (https://moose.ncbs.res.in). We used a D1 SPN morphology obtained from the Luebke repository (Goodliffe et al., 2018) on neuromorpho.org (Ascoli et al., 2007). Dendritic spines were modeled both implicitly and explicitly. Explicit dendritic spines were modeled for synaptic inputs and calcium dynamics at a density of $0.1 \mathrm{spines} / \mu \mathrm{m}$ with cylindrical head $(0.5 \mu \mathrm{m}$ diameter, $0.5 \mu \mathrm{m}$ length) and neck ( $0.12 \mu \mathrm{m}$ diameter, $0.5 \mu \mathrm{m}$ length) on dendritic branches greater than 25 $\mu \mathrm{m}$ from the soma. The density of explicitly modeled spines is not representative of the full spine density observed experimentally, and modeling the full spine density would be computationally intensive. Therefore, the implicit effect of dendritic spines (those not explicitly modeled) on passive membrane properties and dendritic channel densities was modeled by compensating dendritic membrane resistivity $(R M)$, membrane capacitivity $(C M)$, and axial resistivity $(R A)$, as well as channel maximal conductance values (Holmes et al., 2006) using a distance dependent function fit to experimentally observed spine density versus distance from the soma (Wilson, 1992). Values for $R M, C M$, and $R A$ were set to $6.02 \Omega \mathrm{m}^{2}, 0.011 \mathrm{~F} / \mathrm{m}^{2}$, and $1.3 \Omega \mathrm{m}$ respectively, based on automatic parameter optimization.

\section{Voltage gated ion channels}

As previously described (Dorman et al., 2018), the model incorporates the following voltage gated sodium and potassium ion channels that have been observed in SPNs: a fast sodium channel (NaF) (Ogata and Tatebayashi, 1990); fast (Kaf/Kv4.2) (Tkatch et al., 2000) and slow (Kas/Kv1.2) (Shen et al., 2004) A-type potassium channels; an inwardly rectifying potassium channel (Kir2) (Steephen and Manchanda, 2009); a resistant persistent potassium channel (Krp; also called delayed rectifier) (Nisenbaum and Wilson, 1995); a big conductance voltage- and calcium-activated potassium channel (BK) (Berkefeld et al., 2006); and a small conductance calcium-activated potassium channel (SK) (Maylie et al., 2004). Six voltage gated calcium channels (VGCCs) are also included: R-type (CaR/Cav2.3) (Brevi et al., 2001; Foehring et al., 2000), N-type (CaN/Cav2.2) (Bargas et al., 1994; Kasai and Neher, 1992; McNaughton and Randall, 1997), two L-type (CaL1.2/Cav1.2 and CaL1.3/Cav1.3) (Bargas et al., 1994; Kasai and Neher, 1992; Tuckwell, 2012), and two T-type (CaT3.2/Cav3.2/a1 H and CaT3.3/Cav3.3/a1I) (McRory et al., 2001). We newly added a calcium activated chloride channel ( $\mathrm{CaCC}$ ) based on the ANO2/TMEM16B channel, which has been observed in SPNs and allows better fit of the AHP waveform (Song et al., 2016; Pifferi et al., 2009). Channel kinetic equations are the same as our previously reported model, but parameters were updated 
during the parameter optimization by allowing half-activation voltages and time constants to be modified by the parameter optimization algorithm.

Model optimization was done using ajustador software Uedrzejewski-Szmek et al., 2018; Dorman and Blackwell, 2021) that was updated to allow variation of channel kinetics, in addition to channel conductances and membrane properties, in order to fit the model response to experimental data for injected current steps. Briefly, the parameter optimization algorithm utilized a covariance matrix adaptation with evolutionary strategy (cma-es) to vary model parameters (Hansen et al., 2019), and a feature-based fitness metric to compute the fitness between model and experimental data based on features such as number of spikes, action potential height, action potential width, spike timing, steady state membrane voltage, depolarization and hyperpolarization time constants, and after-hyperpolarization waveform. Channel conductances and membrane properties were varied within a linear range, while kinetic parameters (half activation voltage and time constant) were varied with a multiplicative parameter between 0.5 and 2 . Optimizations utilized the Neuroscience Gateway Portal (Sivagnanam et al., 2013).

\section{Calcium dynamics}

Intracellular calcium concentration was modeled with diffusion, calcium-binding buffers, and transmembrane calcium pumps. One-dimensional radial diffusion of buffers and calcium was modeled with concentric shells in dendrites, while spine heads and necks implemented one-dimensional axial diffusion in cylindrical slabs connected from the spine neck to the submembrane shell of the dendritic shaft (Anwar et al., 2014). Transmembrane calcium extrusion mechanisms-plasma membrane calcium ATPase (PMCA) in every compartment and sodium-calcium exchanger (NCX) limited to spines-were modeled with Michaelis-Menten kinetics. The calcium-binding buffers included calbindin and calmodulin ( $\mathrm{N}$ and $\mathrm{C}$ terminals), which could diffuse between calcium compartments, and an endogenous immobile buffer (representative of several potential biological mechanisms that buffer calcium without diffusing) (Matthews et al., 2013; Matthews and Dietrich, 2015).

The sources of calcium influx in the model included the voltage gated calcium channels and the NMDAR synaptic channel. Calcium concentration in the submembrane shell was used for calciumdependent channel activation (BK, SK, CaCC) or inactivation (R-, N-, and L-type calcium channels).

\section{Synaptic channels}

Excitatory NMDAR and AMPAR synaptic channels were included on spine heads, and inhibitory $G_{A B A}$ channels were included on the dendritic shaft. Channels were modeled with dual exponential kinetics, and the NMDA channel included voltage-dependent magnesium blocking.

\section{Plasticity rule}

The calcium based plasticity rule used a dual amplitude and duration threshold for spine calcium concentration to determine LTP and LTD, as we previously described Uędrzejewska-Szmek et al., 2017). For LTD, spine calcium had to exceed the amplitude threshold, $T A_{D}$, of $0.33 \mu \mathrm{M}$ for longer than the duration threshold of $28 \mathrm{~ms}$, while for LTP the amplitude threshold, $T A_{P}$, was $0.53 \mu \mathrm{M}$ and the duration threshold was $3.3 \mathrm{~ms}$. Once thresholds were exceeded, the synaptic weights were updated at each time step toward their maximum or minimum. For LTP, while thresholds were exceeded, synaptic weight was increased according to:

$$
w(t)=\min \left\{\gamma_{P \max }, \gamma_{P} \cdot\left(\left[\mathrm{Ca}^{2+}\right]_{s p}(t-1)-T A_{P}\right)\right\} \cdot \sqrt{1-\frac{w(t-1)-w_{\min }}{w_{\max }-w_{\min }}}
$$

where $\gamma_{P}$ is a gain factor for potentiation, $\gamma_{P \max }$ is the maximum allowable gain value, $\left[\mathrm{Ca}^{2+}\right]_{s p}$ is spine calcium concentration, $T A_{P}$ is the amplitude threshold for potentiation, $w_{\max }$ is maximum allowable synaptic weight (2.0), and $w_{\min }$ is minimum allowable synaptic weight (0). Similarly, when 


\section{Synaptic inputs}

452 soma).

\section{Analysis}

LTD thresholds were exceeded, weight was decreased according to:

$$
w(t)=-\min \left\{\gamma_{D \max }, \gamma_{D} \cdot\left(\left[C a^{2+}\right]_{s p}(t-1)-T A_{D}\right)\right\} \cdot \sqrt{\frac{w(t-1)-w_{\min }}{w_{\max }-w_{\min }}}
$$

where $\gamma_{D}$ and $\gamma_{D \max }$ are the gain and maximum allowable gain for depression, and $T A_{D}$ is the amplitude threshold for depression.

In vivo cortical spike trains were obtained from a CRCNS. org repository, consisting of recordings from 25 simultaneously recorded anterior lateral motor cortex pyramidal neurons with 90 repeated trials (Li et all, 2015). A single initial trial of model input consisted of 200 spike trains, which were selected from 22 similar trials (excluding neurons that were inactive within a trial) in order to preserve within-trial correlations between neurons.

To generate controlled trial-to-trial variability of spike timing, the initial trial was repeated 10 times with random jitter of each spike on each repetition. Trial-to-trial variability was limited to standard deviation of spike timing while constraining the same total number of spikes per spike train within each trial. The random jitter was generate from a truncated normal distribution using a standard deviation of 1, 10, 100, or 200 ms (truncated such that no value outside the start time or end time of the trial was selected). Experiments consisted of 10 trials with a single standard deviation. Trials were separated by a 1 second intertrial interval, during which time membrane potential returned to resting potential and spiking activity ceased.

We also implemented a different type of trial-to-trial variability that allows variability of spike rate for individual synapses, but maintains the same spike timing to the neuron as a whole. Variable spike rate was implemented by randomly moving individual spikes from one presynaptic input train to another, with the probability of each spike being moved between 10-100\%. To maintain the distribution of spike counts per trial, the randomly selected target train probability was weighted by the number of spikes of the target trains. This ensured both the overall spike timing pattern to the whole neuron was maintained as well as the distribution of spike counts to synapses, while allowing small variations in spike rate for each synapse from trial to trial.

To demonstrate the robustness of the results to the spatial pattern of synaptic inputs, a single trial was repeated using 1000 different mappings of spike trains to synapses. This preserved the overall instantaneous firing rate to the neuron, and isolated the contribution of spatial patterns.

In addition to excitatory inputs, the neuron also received two types of inhibitory inputs. Inhibitory inputs were constructed from Poisson processes with mean firing rates consistent with both striatal fast spiking interneurons (FSIs) and low threshold spiking interneurons (LTSIs). Inhibitory trains were active for the entire 21 second experiment duration of the 10 repeated trials.

FSI inputs were generated with a mean firing rate of $12 \mathrm{~Hz}$ (Owen et al., 2018) and targeted densely proximally (within 80 microns of the soma), while LTSI inputs were generated with a mean firing rate of $8 \mathrm{~Hz}$ (Sharott et al., 2012) and targeted distally (greater than 80 microns from the

Analysis of simulations used Python3 and the following python packages: Numpy, Scipy, Pandas, Scikit-learn, Statsmodels, and Matplotlib. For analysis relating spatiotemporal input patterns to magnitude and direction of synaptic weight change, we introduce a "weight-change triggered average," which is constructed by grouping synapses per trial into bins based on the value of the weight change following a trial, computing the instantaneous firing rate of the spike train input to each given synapse, and averaging the instantaneous firing rates within each bin of synaptic weight change. This is analogous to a spike-triggered average (Schwartz et al., 2006), but using the continuous valued, trial-level weight change instead of the spike. 
Random forest regression was applied to the instantaneous firing rate to a synapse, the correlation between synapse firing rate and neighboring synapse firing rate, and the distance from spine to soma. Random forest regression applies a non-linear method for predicting the weight change from a set of features, such as pre-synaptic firing rate. The prediction is determined from a set of hierarchical rules, where each rule partitions the weight change based on a single feature. A non-linear method was required because of the non-linear relationship between weight change and pre-synaptic firing. The instantaneous firing rate of direct input was discretized into $1-$ 5 features (time samples), where 1 time sample calculates the mean firing rate for the entire trial, and 5 time samples calculates mean firing frequency for subsequent $200 \mathrm{~ms}$ time intervals. To determine the optimal features for predicting the weight change, a random forest regression was performed for each combination of features. For each regression, the trial-level weight changes were randomly subdivided into a testing set ( $1 / \mathrm{N}$ of the data) and a training set (the remainder of the data); subdividing the data and performing the random forest regression was repeated $\mathrm{N}$ times. Then analysis of variance was used to determine which combination of features best predicted the weight change.

\section{References}

Abraham WC, Robins A. Memory Retention - the Synaptic Stability versus Plasticity Dilemma. Trends in Neurosciences. 2005 Feb; 28(2):73-78. doi: 10.1016/j.tins.2004.12.003.

Anwar H, Roome CJ, Nedelescu H, Chen W, Kuhn B, De Schutter E. Dendritic Diameters Affect the Spatial Variability of Intracellular Calcium Dynamics in Computer Models. Frontiers in Cellular Neuroscience. 2014 Jul; 8:168. doi: 10.3389/fncel.2014.00168.

Ascoli GA, Donohue DE, Halavi M. NeuroMorpho.Org: A Central Resource for Neuronal Morphologies. Journal of Neuroscience. 2007 Aug; 27(35):9247-9251. doi: 10.1523/JNEUROSCI.2055-07.2007.

Bargas J, Howe A, Eberwine J, Cao Y, Surmeier DJ. Cellular and Molecular Characterization of Ca2+ Currents in Acutely Isolated, Adult Rat Neostriatal Neurons. J Neurosci. 1994 Nov; 14(11 Pt 1):6667-6686.

Berkefeld H, Sailer CA, Bildl W, Rohde V, Thumfart JO, Eble S, Klugbauer N, Reisinger E, Bischofberger J, Oliver D, Knaus HG, Schulte U, Fakler B. BKCa-Cav Channel Complexes Mediate Rapid and Localized Ca2+-Activated K+ Signaling. Science (New York, NY). 2006 Oct; 314(5799):615-20. doi: 10.1126/science.1132915.

Berthet P, Lindahl M, Tully PJ, Hellgren-Kotaleski J, Lansner A. Functional Relevance of Different Basal Ganglia Pathways Investigated in a Spiking Model with Reward Dependent Plasticity. Frontiers in Neural Circuits. 2016; 10:53.

Blackwell KT, Dorman DB, Jędrzejewski-Szmek Z. Github; 2021. https://github.com/neurord/moose_nerp/ releases/tag/v2.1, b78279b.

Bocchio M, Nabavi S, Capogna M. Synaptic Plasticity, Engrams, and Network Oscillations in Amygdala Circuits for Storage and Retrieval of Emotional Memories. Neuron. 2017 May; 94(4):731-743. doi: 10.1016/j.neuron.2017.03.022.

Borroto-Escuela DO, Perez De La Mora M, Manger P, Narváez M, Beggiato S, Crespo-Ramírez M, Navarro G, Wydra K, Díaz-Cabiale Z, Rivera A, Ferraro L, Tanganelli S, Filip M, Franco R, Fuxe K. Brain Dopamine Transmission in Health and Parkinson's Disease: Modulation of Synaptic Transmission and Plasticity Through Volume Transmission and Dopamine Heteroreceptors. Frontiers in Synaptic Neuroscience. 2018; 10. doi: 10.3389/fnsyn.2018.00020.

Brandalise F, Carta S, Helmchen F, Lisman J, Gerber U. Dendritic NMDA Spikes Are Necessary for TimingDependent Associative LTP in CA3 Pyramidal Cells. Nature Communications. 2016 Nov; 7:13480. doi: 10.1038/ncomms13480.

Brevi S, de Curtis M, Magistretti J. Pharmacological and Biophysical Characterization of Voltage-Gated Calcium Currents in the Endopiriform Nucleus of the Guinea Pig. J Neurophysiol. 2001 May; 85(5):2076-2087. doi: 10.1152/jn.2001.85.5.2076.

Buonomano DV, Merzenich MM. CORTICAL PLASTICITY: From Synapses to Maps. Annual Review of Neuroscience. 1998; 21(1):149-186. doi: 10.1146/annurev.neuro.21.1.149. 
542

543

544

545

546

547

548

549

550

551

552

553

554

555

556

557

558

559

560

561

562

563

564

565

566

567

568

569

570

571

572

Caporale N, Dan Y. Spike Timing-Dependent Plasticity: A Hebbian Learning Rule. Annual Review of Neuroscience. 2008; 31(1):25-46. doi: 10.1146/annurev.neuro.31.060407.125639.

Carter AG, Soler-Llavina GJ, Sabatini BL. Timing and Location of Synaptic Inputs Determine Modes of Subthreshold Integration in Striatal Medium Spiny Neurons. The Journal of neuroscience : the official journal of the Society for Neuroscience. 2007 Aug; 27(33):8967-77. doi: 10.1523/JNEUROSCI.2798-07.2007.

Cichon J, Gan WB. Branch-Specific Dendritic Ca2+ Spikes Cause Persistent Synaptic Plasticity. Nature. 2015 Apr; 520(7546):180-185. doi: 10.1038/nature14251.

Cui Y, Prokin I, Mendes A, Berry H, Venance L. Robustness of STDP to Spike Timing Jitter. Scientific Reports. 2018 May; 8(1):8139. doi: 10.1038/s41598-018-26436-y.

Dorman DB, Blackwell KT. Github; 2021. https://github.com/neurord/ajustador/releases/tag/v2.1, d369c05.

Dorman DB, Jędrzejewska-Szmek J, Blackwell KT. Inhibition Enhances Spatially-Specific Calcium Encoding of Synaptic Input Patterns in a Biologically Constrained Model. eLife. 2018 Oct; 7:e38588. doi: 10.7554/eLife.38588.

Du K, Wu YW, Lindroos R, Liu Y, Rózsa B, Katona G, Ding JB, Kotaleski JH. Cell-Type-Specific Inhibition of the Dendritic Plateau Potential in Striatal Spiny Projection Neurons. Proceedings of the National Academy of Sciences. 2017 Sep; 114(36):E7612-E7621. doi: 10.1073/pnas.1704893114.

Dunovan K, Vich C, Clapp M, Verstynen T, Rubin J. Reward-driven changes in striatal pathway competition shape evidence evaluation in decision-making. PLoS Comput Biol. 2019; 15(5):e1006998.

El-Boustani S, Ip JPK, Breton-Provencher V, Knott GW, Okuno H, Bito H, Sur M. Locally Coordinated Synaptic Plasticity of Visual Cortex Neurons in Vivo. Science. 2018 Jun; 360(6395):1349-1354. doi: 10.1126/science.aao0862.

Evans RC, Blackwell KT. Calcium: Amplitude, Duration, or Location? Biological Bulletin. 2015; 228(1):75-83. doi: 10.1086/BBLv228n1p75.

Faisal AA, Selen LPJ, Wolpert DM. Noise in the Nervous System. Nature Reviews Neuroscience. 2008 Apr; 9(4):292-303. doi: 10.1038/nrn2258.

Fisher SD, Robertson PB, Black MJ, Redgrave P, Sagar MA, Abraham WC, Reynolds JNJ. Reinforcement Determines the Timing Dependence of Corticostriatal Synaptic Plasticity in Vivo. Nature Communications. 2017 Dec; 8(1):334. doi: 10.1038/s41467-017-00394-x.

Foehring RC, Mermelstein PG, Song WJ, Ulrich S, Surmeier DJ. Unique Properties of R-Type Calcium Currents in Neocortical and Neostriatal Neurons. Journal of neurophysiology. 2000 Nov; 84(5):2225-2236.

Golding NL, Staff NP, Spruston N. Dendritic Spikes as a Mechanism for Cooperative Long-Term Potentiation. Nature. 2002 Jul; 418(6895):326-331. doi: 10.1038/nature00854.

Goodliffe JW, Song H, Rubakovic A, Chang W, Medalla M, Weaver CM, Luebke JI. Differential Changes to D1 and D2 Medium Spiny Neurons in the 12-Month-Old Q175+/- Mouse Model of Huntington's Disease. PLOS ONE. 2018 Aug; 13(8):e0200626. doi: 10.1371/journal.pone.0200626.

Gordon U, Polsky A, Schiller J. Plasticity Compartments in Basal Dendrites of Neocortical Pyramidal Neurons. Journal of Neuroscience. 2006 Dec; 26(49):12717-12726. doi: 10.1523/JNEUROSCI.3502-06.2006.

Govindarajan A, Israely I, Huang SY, Tonegawa S. The Dendritic Branch Is the Preferred Integrative Unit for Protein Synthesis-Dependent LTP. Neuron. 2011 Jan; 69(1):132-146. doi: 10.1016/j.neuron.2010.12.008.

Graupner M, Brunel N. Calcium-Based Plasticity Model Explains Sensitivity of Synaptic Changes to Spike Pattern, Rate, and Dendritic Location. Proceedings of the National Academy of Sciences. 2012 Mar; 109(10):3991-3996. doi: 10.1073/pnas.1109359109.

Graupner M, Wallisch P, Ostojic S. Natural Firing Patterns Imply Low Sensitivity of Synaptic Plasticity to Spike Timing Compared with Firing Rate. Journal of Neuroscience. 2016; 36(44):11238-11258. doi: 10.1523/JNEUROSCI.0104-16.2016.

Hansen N, Akimoto Y, Baudis P. CMA-ES/Pycma on Github. Zenodo. 2019 Feb; doi: 10.5281/zenodo.2559634.

Hawes SL, Gillani F, Evans RC, Benkert EA, Blackwell KT. Sensitivity to Theta-Burst Timing Permits LTP in Dorsal Striatal Adult Brain Slice. Journal of neurophysiology. 2013 Nov; 110(9):2027-36. doi: 10.1152/jn.00115.2013. 
He K, Huertas M, Hong SZ, Tie X, Hell JW, Shouval H, Kirkwood A. Distinct Eligibility Traces for LTP and LTD in Cortical Synapses. Neuron. 2015 Nov; 88(3):528-538. doi: 10.1016/j.neuron.2015.09.037.

Holmes WR, Ambros-Ingerson J, Grover LM. Fitting Experimental Data to Models That Use Morphological Data from Public Databases. Journal of Computational Neuroscience. 2006 Jun; 20(3):349-365. doi: 10.1007/s10827-006-7189-8.

Jędrzejewski-Szmek Z, Abrahao KP, Jędrzejewska-Szmek J, Lovinger DM, Blackwell KT. Parameter Optimization Using Covariance Matrix Adaptation-Evolutionary Strategy (CMA-ES), an Approach to Investigate Differences in Channel Properties Between Neuron Subtypes. Frontiers in Neuroinformatics. 2018; 12. doi: 10.3389/fninf.2018.00047.

Jędrzejewska-Szmek J, Damodaran S, Dorman DB, Blackwell KT. Calcium Dynamics Predict Direction of Synaptic Plasticity in Striatal Spiny Projection Neurons. European Journal of Neuroscience. 2017 Apr; 45(8):10441056. doi: 10.1111/ejn.13287.

Josselyn SA, Tonegawa S. Memory Engrams: Recalling the Past and Imagining the Future. Science. 2020 Jan; 367(6473). doi: 10.1126/science.aaw4325.

Kasai H, Neher E. Dihydropyridine-Sensitive and Omega-Conotoxin-Sensitive Calcium Channels in a Mammalian Neuroblastoma-Glioma Cell Line. The Journal of Physiology. 1992 Mar; 448(1):161-188. doi: 10.1113/jphysiol.1992.sp019035.

Kastellakis G, Cai DJ, Mednick SC, Silva AJ, Poirazi P. Synaptic Clustering within Dendrites: An Emerging Theory of Memory Formation. Progress in neurobiology. 2015 Mar; 126:19-35. doi: 10.1016/j.pneurobio.2014.12.002.

Kerlin A, Mohar B, Flickinger D, MacLennan BJ, Davis C, Spruston N, Svoboda K. Functional Clustering of Dendritic Activity during Decision-Making. bioRxiv. 2018 Oct; p. 440396. doi: 10.1101/440396.

Kreitzer AC, Malenka RC. Striatal Plasticity and Basal Ganglia Circuit Function. Neuron. 2008 Nov; 60(4):543-54. doi: 10.1016/j.neuron.2008.11.005.

Lappalainen J, Herpich J, MTetzlaff C. A Theoretical Framework to Derive Simple, Firing-Rate-Dependent Mathematical Models of Synaptic Plasticity. Front Comput Neurosci. 2019; 13(38):26.

Larkum ME, Nevian T, Sandler M, Polsky A, Schiller J. Synaptic Integration in Tuft Dendrites of Layer 5 Pyramidal Neurons: A New Unifying Principle. Science (New York, NY). 2009 Aug; 325(5941):756-60. doi: 10.1126/science.1171958.

Legenstein R, Maass W. Branch-Specific Plasticity Enables Self-Organization of Nonlinear Computation in Single Neurons. The Journal of neuroscience : the official journal of the Society for Neuroscience. 2011 Jul; 31(30):10787-802. doi: 10.1523/JNEUROSCI.5684-10.2011.

Legenstein R, Naeger C, Maass W. What Can a Neuron Learn with Spike-Timing-Dependent Plasticity? Neural Computation. 2005 Nov; 17(11):2337-2382. doi: 10.1162/0899766054796888.

Li N, Chen TW, Guo ZV, Gerfen CR, Svoboda K. A Motor Cortex Circuit for Motor Planning and Movement. Nature. 2015 Mar; 519(7541):51-56. doi: 10.1038/nature14178.

Losonczy A, Makara JK, Magee JC. Compartmentalized Dendritic Plasticity and Input Feature Storage in Neurons. Nature. 2008 Mar; 452(7186):436-441. doi: 10.1038/nature06725.

Lovinger DM, Tyler EC, Merritt A. Short- and Long-Term Synaptic Depression in Rat Neostriatum. Journal of Neurophysiology. 1993 Nov; 70(5):1937-1949. doi: 10.1152/jn.1993.70.5.1937.

Magó Á, Weber JP, Ujfalussy BB, Makara JK. Synaptic Plasticity Depends on the Fine-Scale Input Pattern in Thin Dendrites of CA1 Pyramidal Neurons. Journal of Neuroscience. 2020 Mar; 40(13):2593-2605. doi: 10.1523/JNEUROSCI.2071-19.2020.

Makara JK, Magee JC. Variable Dendritic Integration in Hippocampal CA3 Pyramidal Neurons. Neuron. 2013 Dec; 80(6):1438-50. doi: 10.1016/j.neuron.2013.10.033.

Martin SJ, Morris RGM. New Life in an Old Idea: The Synaptic Plasticity and Memory Hypothesis Revisited. Hippocampus. 2002; 12(5):609-636. doi: 10.1002/hipo.10107. 
Matthews EA, Dietrich D. Buffer Mobility and the Regulation of Neuronal Calcium Domains. Frontiers in Cellular Neuroscience. 2015 Feb; 9:48. doi: 10.3389/fncel.2015.00048.

Matthews EA, Schoch S, Dietrich D. Tuning Local Calcium Availability: Cell-Type-Specific Immobile Calcium Buffer Capacity in Hippocampal Neurons. Journal of Neuroscience. 2013 Sep; 33(36):14431-14445. doi: 10.1523/JNEUROSCI.4118-12.2013.

Maylie J, Bond CT, Herson PS, Lee WS, Adelman JP. Small Conductance Ca 2+ -Activated K + Channels and Calmodulin. The Journal of Physiology. 2004 Jan; 554(2):255-261. doi: 10.1113/jphysiol.2003.049072.

McNaughton NCL, Randall AD. Electrophysiological Properties of the Human N-Type Ca2+ Channel: I. Channel Gating in $\mathrm{Ca} 2+\mathrm{Ba} 2+$ and Sr2+ Containing Solutions. Neuropharmacology. 1997 Jul; 36(7):895-915. doi: 10.1016/S0028-3908(97)00085-3.

McRory JE, Santi CM, Hamming KSC, Mezeyova J, Sutton KG, Baillie DL, Stea A, Snutch TP. Molecular and Functional Characterization of a Family of Rat Brain T-Type Calcium Channels. Journal of Biological Chemistry. 2001 Feb; 276(6):3999-4011. doi: 10.1074/jbc.M008215200.

Nevian T, Sakmann B. Spine Ca2+ Signaling in Spike-Timing-Dependent Plasticity. Journal of Neuroscience. 2006 Oct; 26(43):11001-11013. doi: 10.1523/JNEUROSCI.1749-06.2006.

Nisenbaum ES, Wilson CJ. Potassium Currents Responsible for Inward and Outward Rectification in Rat Neostriatal Spiny Projection Neurons. The Journal of neuroscience : the official journal of the Society for Neuroscience. 1995 Jun; 15(6):4449-63.

Ogata N, Tatebayashi H. Sodium Current Kinetics in Freshly Isolated Neostriatal Neurones of the Adult Guinea Pig. Pflugers Archiv : European journal of physiology. 1990 Jul; 416(5):594-603. doi: 10.1007/BF00382695.

Oh WC, Parajuli LK, Zito K. Heterosynaptic Structural Plasticity on Local Dendritic Segments of Hippocampal CA1 Neurons. Cell Reports. 2015 Jan; 10(2):162-169. doi: 10.1016/j.celrep.2014.12.016.

Oikonomou KD, Singh MB, Sterjanaj EV, Antic SD. Spiny Neurons of Amygdala, Striatum, and Cortex Use Dendritic Plateau Potentials to Detect Network UP States. Frontiers in Cellular Neuroscience. 2014 Sep; 8:292. doi: 10.3389/fncel.2014.00292.

Owen SF, Berke JD, Kreitzer AC. Fast-spiking interneurons supply feedforward control of bursting, calcium, and plasticity for efficient learning. Cell. 2018; 172(4):683-695.

Perrin E, Venance L. Bridging the Gap between Striatal Plasticity and Learning. Current Opinion in Neurobiology. 2019 Feb; 54:104-112. doi: 10.1016/j.conb.2018.09.007.

Pifferi S, Dibattista M, Menini A. TMEM16B Induces Chloride Currents Activated by Calcium in Mammalian Cells. Pflügers Archiv - European Journal of Physiology. 2009 Oct; 458(6):1023-1038. doi: 10.1007/s00424009-0684-9.

Plotkin JL, Day M, Surmeier DJ. Synaptically Driven State Transitions in Distal Dendrites of Striatal Spiny Neurons. Nature neuroscience. 2011 Jun; 14(7):881-8. doi: 10.1038/nn.2848.

Prager EM, Dorman DB, Hobel ZB, Malgady JM, Blackwell KT, Plotkin JL. Dopamine Oppositely Modulates State Transitions in Striosome and Matrix Direct Pathway Striatal Spiny Neurons. Neuron. 2020 Dec; 108(6):10911102.e5. doi: 10.1016/j.neuron.2020.09.028.

van Rossum MCW, Bi GQ, Turrigiano GG. Stable Hebbian Learning from Spike Timing-Dependent Plasticity. Journal of Neuroscience. 2000 Dec; 20(23):8812-8821. doi: 10.1523/JNEUROSCI.20-23-08812.2000.

Santos FJ, Oliveira RF, Jin X, Costa RM. Corticostriatal Dynamics Encode the Refinement of Specific Behavioral Variability during Skill Learning. eLife. 2015 Sep; 4:e09423. doi: 10.7554/eLife.09423.

Schiller J, Major G, Koester HJ, Schiller Y. NMDA Spikes in Basal Dendrites of Cortical Pyramidal Neurons. Nature. 2000 Mar; 404(6775):285-9. doi: 10.1038/35005094.

Schwartz O, Pillow JW, Rust NC, Simoncelli EP. Spike-Triggered Neural Characterization. Journal of Vision. 2006 Feb; 6(4):13-13. doi: 10.1167/6.4.13.

Shadlen MN, Newsome WT. The Variable Discharge of Cortical Neurons: Implications for Connectivity, Computation, and Information Coding. Journal of Neuroscience. 1998 May; 18(10):3870-3896. doi: 10.1523/JNEUROSCI.18-10-03870.1998. 
Sharott A, Doig NM, Mallet N, Magill PJ. Relationships between the firing of identified striatal interneurons and spontaneous and driven cortical activities in vivo. Journal of Neuroscience. 2012; 32(38):13221-13236.

Shen W, Hernandez-Lopez S, Tkatch T, Held JE, Surmeier DJ. Kv1.2-Containing K+ Channels Regulate Subthreshold Excitability of Striatal Medium Spiny Neurons. Journal of neurophysiology. 2004 Mar; 91(3):1337-49. doi: 10.1152/jn.00414.2003.

Shindou T, Shindou M, Watanabe S, Wickens J. A Silent Eligibility Trace Enables Dopamine-Dependent Synaptic Plasticity for Reinforcement Learning in the Mouse Striatum. European Journal of Neuroscience. 2019; 49(5):726-736. doi: 10.1111/ejn.13921.

Sivagnanam S, Majumdar A, Yoshimoto K, Astakhov V, Bandrowski AE, Martone ME, Carnevale NT. Introducing the Neuroscience Gateway. IWSG. 2013; 993.

Sjöström PJ, Rancz EA, Roth A, Häusser M. Dendritic Excitability and Synaptic Plasticity. Physiological Reviews. 2008 Apr; 88(2):769-840. doi: 10.1152/physrev.00016.2007.

Sjöström PJ, Turrigiano GG, Nelson SB. Rate, Timing, and Cooperativity Jointly Determine Cortical Synaptic Plasticity. Neuron. 2001 Dec; 32(6):1149-1164. doi: 10.1016/S0896-6273(01)00542-6.

Song SC, Beatty JA, Wilson CJ. The Ionic Mechanism of Membrane Potential Oscillations and Membrane Resonance in Striatal LTS Interneurons. Journal of Neurophysiology. 2016; 116(4).

Steephen JE, Manchanda R. Differences in Biophysical Properties of Nucleus Accumbens Medium Spiny Neurons Emerging from Inactivation of Inward Rectifying Potassium Currents. Journal of Computational Neuroscience. 2009 Dec; 27(3):453-470. doi: 10.1007/s10827-009-0161-7.

Stevens CF, Zador AM. Input Synchrony and the Irregular Firing of Cortical Neurons. Nature Neuroscience. 1998 Oct; 1(3):210-217. doi: 10.1038/659.

Takahashi N, Kitamura K, Matsuo N, Mayford M, Kano M, Matsuki N, Ikegaya Y. Locally Synchronized Synaptic Inputs. Science. 2012; 335(6066):353-356. doi: 10.1126/science.1210362.

Takeuchi T, Duszkiewicz A, Morris R. The Synaptic Plasticity and Memory Hypothesis: Encoding, Storage and Persistence. Philosophical transactions of the Royal Society of London Series B, Biological sciences. 2014 Jan; 369:20130288. doi: 10.1098/rstb.2013.0288.

Tkatch T, Baranauskas G, Surmeier DJ. Kv4.2 mRNA Abundance and A-Type K(+) Current Amplitude Are Linearly Related in Basal Ganglia and Basal Forebrain Neurons. The Journal of neuroscience : the official journal of the Society for Neuroscience. 2000 Jan; 20(2):579-88.

Trachtenberg JT, Chen BE, Knott GW, Feng G, Sanes JR, Welker E, Svoboda K. Long-Term in Vivo Imaging of Experience-Dependent Synaptic Plasticity in Adult Cortex. Nature. 2002 Dec; 420(6917):788-794. doi: 10.1038/nature01273.

Tuckwell HC. Quantitative Aspects of L-Type Ca2+ Currents. Progress in neurobiology. 2012 Jan; 96(1):1-31. doi: 10.1016/j.pneurobio.2011.09.010.

Ujfalussy BB, Makara JK. Impact of Functional Synapse Clusters on Neuronal Response Selectivity. Nature Communications. 2020 Mar; 11(1):1413. doi: 10.1038/s41467-020-15147-6.

Weber JP, Andrásfalvy BK, Polito M, Magó Á, Ujfalussy BB, Makara JK. Location-Dependent Synaptic Plasticity Rules by Dendritic Spine Cooperativity. Nature Communications. 2016 Apr; 7:11380. doi: 10.1038/ncomms11380.

Williams AH, Poole B, Maheswaranathan N, Dhawale AK, Fisher T, Wilson CD, Brann DH, Trautmann EM, Ryu S, Shusterman R, Rinberg D, Ölveczky BP, Shenoy KV, Ganguli S. Discovering Precise Temporal Patterns in Large-Scale Neural Recordings through Robust and Interpretable Time Warping. Neuron. 2019 Nov; doi: 10.1016/j.neuron.2019.10.020.

Wilson CJ. Dendritic Morphology, Inward Rectification, and the Functional Properties of Neostriatal Neurons. In: Single Neuron Computation Elsevier; 1992.p. 141-171. doi: 10.1016/B978-0-12-484815-3.50012-8.

Wilson DE, Whitney DE, Scholl B, Fitzpatrick D. Orientation Selectivity and the Functional Clustering of Synaptic Inputs in Primary Visual Cortex. Nature Neuroscience. 2016 Jun; 19(8):1003-1009. doi: 10.1038/nn.4323. 
732 Winnubst J, Cheyne JE, Niculescu D, Lohmann C. Spontaneous Activity Drives Local Synaptic Plasticity In Vivo.

$733 \quad$ Neuron. 2015; 87(2):399-410. doi: 10.1016/j.neuron.2015.06.029.

Yagishita S, Hayashi-Takagi A, Ellis-Davies GCR, Urakubo H, Ishii S, Kasai H. A Critical Time Window for 735 Dopamine Actions on the Structural Plasticity of Dendritic Spines. Science. 2014 Sep; 345(6204):1616-1620.

736 doi: 10.1126/science.1255514.

737 Zhang Y, Cudmore RH, Lin DT, Linden DJ, Huganir RL. Visualization of NMDA Receptor-Dependent AMPA Re738 ceptor Synaptic Plasticity in Vivo. Nature Neuroscience. 2015 Mar; 18(3):402-407. doi: 10.1038/nn.3936.

739 Zoli M, Torri C, Ferrari R, Jansson A, Zini I, Fuxe K, Agnati LF. The Emergence of the Volume Transmission $740 \quad$ Concept. Brain Research Reviews. 1998 May; 26(2):136-147. doi: 10.1016/S0165-0173(97)00048-9.

741 Zucker RS. Calcium- and Activity-Dependent Synaptic Plasticity. Current opinion in neurobiology. 1999 Jun; 742 9(3):305-13. doi: 10.1016/S0959-4388(99)80045-2. 